\title{
Bounded Solutions of Some Nonlinear Elliptic Equations in Cylindrical Domains
}

\author{
Angel Calsina ${ }^{1}$, Joan Solà-Morales ${ }^{2}$ and Marta València ${ }^{3}$
}

\section{Abstract.}

The existence of a (unique) solution of the second order semilinear elliptic equation

$$
\sum_{i, j=0}^{n} a_{i j}(x) u_{x_{i} x_{j}}+f(\nabla u, u, x)=0
$$

with $x=\left(x_{0}, x_{1}, \ldots, x_{n}\right) \in\left(s_{0}, \infty\right) \times \Omega^{\prime}$, for a bounded domain $\Omega^{\prime}$, together with the additional conditions

$$
\begin{aligned}
& u(x)=0 \text { for }\left(x_{1}, x_{2}, \ldots, x_{n}\right) \in \partial \Omega^{\prime} \\
& u(x)=\varphi\left(x_{1}, x_{2}, \ldots, x_{n}\right) \text { for } x_{0}=s_{0} \\
& |u(x)| \quad \text { globally bounded }
\end{aligned}
$$

is shown to be a well posed problem under some sign and growth restrictions on $f$ and its partial derivatives. It can be seen as an initial value problem, with initial value $\varphi$, in the space $\mathcal{C}_{0}^{0}\left(\overline{\Omega^{\prime}}\right)$ and satisfying the strong order-preserving property. In the case that $a_{i j}$ and $f$ do not depend on $x_{0}$ or are periodic in $x_{0}$ it is shown that the corresponding dynamical system has a compact global attractor. Also, conditions on $f$ are given under which all the solutions tend to zero as $x_{0}$ tends to infinity. Proofs are strongly based on maximum and comparison techniques.

\section{Introduction}

In many contexts, boundary-value problems for elliptic equations in unbounded domains include the condition that the solutions have to assume some given values at infinity, these values being data of the problem, usually supported by physical experience. In view of this it seems interesting to impose at infinity only the condition that the solutions have to be bounded and then to ask oneself the question of what are the possible behaviours at infinity of these bounded solutions.

Of course this is a difficult problem for a general equation. It is similar in nature to the problem of finding the global attractor of a Dynamical System, because compact global attractors, when they exist, contain the different kinds of behaviour at infinity (in time) that can be expected.

Our paper goes in this direction, that is of exploiting this similarity. More precisely we obtain conditions for the bounded solutions of a semilinear elliptic problem in a cylindrical domain to define a Dynamical System in a suitable Banach Space where the coordinate along the axis of the cylinder plays the role of time.

\footnotetext{
${ }^{1}$ Departament de Matemàtiques, Facultat de Ciències, UAB. 08193-Bellaterra, Barcelona. Spain.

${ }^{2}$ Departament de Matemàtica Aplicada I - UPC. Diagonal, 647. 08028-Barcelona. Spain.

${ }^{3}$ Departament de Matemàtica Aplicada I - UPC. Diagonal, 647. 08028-Barcelona. Spain.
} 
Much work on asymptotic behaviour for the solutions of the problems we consider can be done without appeal to the formalism or the theory of Dynamical Systems, and we mention the papers $[\mathbf{1}],[\mathbf{6}]$ and $[\mathbf{1 2}]$ as some recent contributions that do not use this approach, and [5] for results in more general unbounded domains. But a Dynamical Systems approach, either global or local, has also been successfully used in $[\mathbf{2}],[\mathbf{3}],[\mathbf{4}],[\mathbf{9}]$ and $[\mathbf{1 1}]$, for example.

We will consider semilinear second order elliptic equations of the form

$$
\sum_{i, j=0}^{n} a_{i j}(x) u_{x_{i} x_{j}}+f(\nabla u, u, x)=0
$$

for $x=\left(x_{0}, x_{1}, \ldots, x_{n}\right)$ in a cylindrical domain of the form $\Omega=\left(s_{0}, \infty\right) \times \Omega^{\prime}$ with $\Omega^{\prime}$ a smooth bounded domain of $\mathbf{R}^{n}$, together with the additional conditions

$$
\begin{array}{cl}
u(x)=0 & \text { for } \quad\left(x_{1}, x_{2}, \ldots, x_{n}\right) \in \partial \Omega^{\prime} \\
|u(x)| & \text { globally bounded }
\end{array}
$$

We are willing to see the problem of existence of a solution of (1.1)-(1.3) with a given "initial" value

$$
u(x)=\varphi\left(x_{1}, x_{2}, \ldots, x_{n}\right) \text { for } \quad x_{0}=s_{0}
$$

as a well posed initial value problem.

The fact that we take the cylinder to be $\left(s_{0}, \infty\right) \times \Omega^{\prime}$ instead of $(-\infty, \infty) \times \Omega^{\prime}$ is in agreement with other evolution equations where the solutions exist forwards in time but need not to exist backwards. Nevertheless, for the case where (1.1) does not depend on $x_{0}$ or is periodic in $x_{0}$ and under the conditions we will give in Section 3 in order to have a dynamical system with a compact global attractor, this attractor will be made exactly of the solutions that exist and are bounded in $-\infty<x_{0}<\infty$.

This kind of solutions have an important physical meaning when the corresponding elliptic equation is that satisfied by travelling wave solutions of a parabolic reaction-diffusion equation in $(-\infty, \infty) \times \Omega^{\prime}$. So, the set of all travelling wave solutions of a given wave-speed can be characterized, at least for some nonlinearities and some wave-speeds, as the set of orbits of the attractor of the dynamical system that we obtain.

Our paper is very much motivated by [3] but wants to be an alternative approach to some parts of it in order to improve certain results and to clarify some restrictions. The main difference is in the extensive use here of the maximum principle and the classical methods of the Theory of Partial Differential Equations, in comparison with the abstract-semigroup approach considered there. In particular this means that we will work with classical solutions in spaces of continuous functions instead of the solutions in Sobolev spaces used there.

In our case we are able to obtain a dynamical system even when the nonlinearity includes gradient terms. It is not clear if this can be done with the methods of [3]. In [3] (Theorem 7.1) bounded solutions were obtained, by invariant manifold 
techniques, among all the solutions. So, restrictions were imposed to the Lipschitz constant of the nonlinearity for these manifolds to exist. In the present case our restrictions are only one sided $(\operatorname{sign})$ conditions: roughly speaking they are upper bounds on $\partial f / \partial u$ instead of on $|\partial f / \partial u|$. More precisely we ask for a balance between the reaction term $\partial f / \partial u$ and the convection term $\partial f / \partial u_{x_{0}}$. See Proposition 2.1 and Proposition 2.2 of the present paper for more details.

Also, in comparison with $[\mathbf{3}]$, the use of the maximum principle here restricts ourselves to second order scalar equations that are elliptic in the classical sense. But in counterpart we can deal with more general nonlinearities.

The paper is organized as follows: In Section 2 we study the existence and uniqueness of solutions of the problem (1.1)-(1.4) that can be seen as a kind of initial boundary value problem, in some sense similar to parabolic problems. Nevertheless, the global condition (1.3) makes a big difference, in the sense that it is not possible to speak of local solutions and then use continuation arguments, but the global solutions have to be obtained from the beginning.

Existence, uniqueness and also continuity with respect to the initial state are obtained with the help of the maximum principle. Proposition 2.1 and Proposition 2.2 give sufficient conditions on the nonlinearity $f$ for that principle to be applicable. It needs to be emphasized that these sufficient conditions represent an important restriction on the nonlinearity: only nonlinearities with large downstream convection (large negative $\partial f / \partial u_{x_{0}}$ in comparison with $\partial f / \partial u$ ) produce a well posed initial value problem for (1.1)-(1.4). Theorem 2.3 and Theorem 2.4 give precise statements of existence, uniqueness, regularity and continuity. Theorem 2.7 gives the property that the problem is strongly order-preserving.

We remark that with not much changes the statements and the proofs also hold for a particular case of the quasilinear version of equation (1.1), namely when the coefficient functions $a_{i j}$ depend on $x$ and also on $\nabla u$ but not on $u_{x_{0}}$.

Section 3 is devoted to the global properties of the dynamical system defined by the initial value problem considered in Section 2. The Dynamical Systems approach is only possible when the $a_{i j}(x)$ and $f$ do not depend on $x_{0}$ (autonomous equation) or are periodic in this variable. In both cases, under the same conditions that were used to prove existence of solutions, it is proven in Theorem 3.5 and Theorem 3.6 that the system has a connected compact global attractor. According to the results of $[\boldsymbol{8}]$ this is a consequence of the fact that the dynamical system turns out to be completely continuous and dissipative (i.e. there is a bounded set that attracts every orbit of the system). The proof of dissipativeness does not follow the most common arguments used when dealing with parabolic or hyperbolic problems (Lyapunov functions) but an idea based on the maximum principle. This is stated as an abstract criterion for dissipativeness in Proposition 3.3 and Proposition 3.4 and used in the proofs of Theorem 3.5 and Theorem 3.6.

As we said above this attractor summarizes in some sense all the possible asymptotic behaviours when $x_{0}$ tends to $\infty$ of the bounded solutions of the elliptic problem. It consists exactly of the solutions existing in the whole range $-\infty<x_{0}<\infty$ and being globally bounded, a kind of solution of special relevance, for example when looking for travelling wave solutions of parabolic equations.

Section 4 deals with convergence of all the bounded solutions to zero. In Theorem 4.5 one sees that under the sign conditions on $f$ (those of a restoring force) 
that more or less could be expected, all the solutions tend to zero. Nevertheless in Theorem 4.6 and Theorem 4.7 one sees that the sign conditions on $f$ of Theorem 4.5 can be relaxed in some particular situations and one also obtains convergence to zero. When these conditions are relaxed they still take also into account the convection term. In the autonomous and periodic cases these three theorems imply that the attractor reduces to zero.

Of course, the results of Section 4 should not be interpreted as an indication that the attractors obtained in Section 3 are most likely reduced to a single point. As an example of non-trivial attractor one can take $\Omega^{\prime}=(0,1)$ and the equation

$$
u_{x_{0} x_{0}}+u_{x_{1} x_{1}}+B_{0} u_{x_{0}}+C u-u^{3}=0
$$

for large $C$ and $B_{0}<-2 \sqrt{C}$. As it will be seen below in the example after Proposition 2.2 this equation satisfies the hypotheses required to define a dynamical system with a compact global attractor. It is known that the two-point boundary value problem $u^{\prime \prime}+C u-u^{3}=0, u(0)=u(1)=0$, has several nontrivial solutions for large $C$. The attractor is a connected set containing at least these equilibria.

Let us make a final remark about notation. We will use independently the notation $x=\left(x_{0}, x_{1}, \ldots, x_{n}\right)$ for a point in $\bar{\Omega}=\left[s_{0}, \infty\right) \times \overline{\Omega^{\prime}} \subset \mathbf{R} \times \mathbf{R}^{n}$ or the notation $x=(s, y)$ with $s \in\left[s_{0}, \infty\right)$ and $y=\left(x_{1}, x_{2}, \ldots, x_{n}\right) \in \overline{\Omega^{\prime}}$. The first notation $x=\left(x_{0}, \ldots, x_{n}\right)$ will be useful for the approach to the problem as a classical elliptic equation, and the notation $x=(s, y)$ will emphasize that the variable $s$ plays the role of time in the domain $\bar{\Omega}=\left[s_{0}, \infty\right) \times \overline{\Omega^{\prime}}$.

We will use the notation $\mathcal{C}_{0}^{\alpha}\left(\overline{\Omega^{\prime}}\right)$ to mean the space of $\mathcal{C}^{\alpha}$-functions in $\overline{\Omega^{\prime}}$ that vanish on $\partial \Omega^{\prime}$.

\section{Existence of solutions}

Let $S$ be a semilinear elliptic operator of the form

$$
S[u]=\sum_{i, j=0}^{n} a_{i j}(x) u_{x_{i} x_{j}}+f(\nabla u, u, x)
$$

defined for $u$ as a function of $x=\left(x_{0}, x_{1}, \ldots, x_{n}\right) \in \Omega=\left(s_{0}, \infty\right) \times \Omega^{\prime} \subset \mathbf{R}^{n+1}$, where $\Omega^{\prime}$ is a bounded domain in $\mathbf{R}^{n}$.

We assume the following hypothesis concerning ellipticity and smoothness of $S$ and $\Omega$ :

H1. The boundary $\partial \Omega^{\prime}$ is of class $\mathcal{C}^{2+\gamma}$ for some $\gamma \in(0,1)$. The functions $a_{i j}(x)$ are of class $\mathcal{C}^{1+\gamma}$ in $\bar{\Omega}=\left[s_{0}, \infty\right) \times \overline{\Omega^{\prime}}$ for $i, j=0,1, \ldots, n$, and there exists a continuous function $\nu:\left[s_{0}, \infty\right) \longrightarrow(0, \infty)$ such that the inequality

$$
\sum_{i, j=0}^{n} a_{i j}(x) \xi_{i} \xi_{j} \geq \nu\left(x_{0}\right) \sum_{i=0}^{n} \xi_{i}^{2}
$$


holds for all $x \in \bar{\Omega}$ and $\left(\xi_{0}, \ldots, \xi_{n}\right) \in \mathbf{R}^{n+1}$. The nonlinear function $f(p, z, x)$ is of class $\mathcal{C}^{1}$ in $\mathbf{R}^{n+1} \times \mathbf{R} \times \bar{\Omega}$.

Remark: Observe that under the hypothesis $\mathrm{H} 1$ the operator $S$ can be written with the principal part in divergence form and the nonlinear part being a function of $\operatorname{class} \mathcal{C}^{\gamma}$.

We also suppose that $S$ satisfies the following hypothesis of weighted strong monotonicity:

H2. There exists a positive continuous weight function $\mu: \bar{\Omega} \rightarrow(0, \infty)$, tending to zero as $x_{0}$ tends to infinity uniformly on $\left(x_{1}, x_{2}, \ldots, x_{n}\right) \in \overline{\Omega^{\prime}}$, such that the following two properties hold for any pair of bounded functions $u, v$ in $\mathcal{C}^{2}\left(\Omega_{0}\right) \cap$ $\mathcal{C}\left(\overline{\Omega_{0}}\right)$, where $\Omega_{0}$ is any domain (bounded or not) contained in $\Omega\left(\Omega_{0} \subseteq \Omega\right)$ :

i) If $S[u]-S[v] \geq 0$ in $\Omega_{0}$, then

$$
\mu(x)(u(x)-v(x)) \leq \max \left\{0, \sup _{\partial \Omega_{0}} \mu(x)(u(x)-v(x))\right\}
$$

for all $x \in \Omega_{0}$, and the inequality is strict unless $\mu(x)(u(x)-v(x))$ is a constant in $\Omega_{0}$.

ii) If $S[u]-S[v] \leq 0$ in $\Omega_{0}$, then

$$
\mu(x)(u(x)-v(x)) \geq \min \left\{0, \inf _{\partial \Omega_{0}} \mu(x)(u(x)-v(x))\right\}
$$

for all $x \in \Omega_{0}$, and the inequality is strict unless $\mu(x)(u(x)-v(x))$ is a constant in $\Omega_{0}$.

Remark: If $S$ was linear hypothesis $\mathrm{H} 2$ would be equivalent to the classical maximum and minimum principles with a weight $\mu(x)$. The fact that $\mu(x)$ tends to zero as $x_{0}$ tends to infinity is useful to deal with bounded solutions in an unbounded domain.

Remark: Observe that if $S$ satisfies hypothesis $\mathrm{H} 2$ and $S[u]=S[v]$ in $\Omega_{0}$, then

$$
|\mu(x)(u(x)-v(x))| \leq \sup _{\partial \Omega_{0}}|\mu(x)(u(x)-v(x))|
$$

for all $x \in \Omega_{0}$, and the inequality is strict unless $\mu(x)(u(x)-v(x))$ is a constant in $\Omega_{0}$.

A sufficient condition for hypothesis $\mathrm{H} 2$ to hold is given by the following proposition that precises the balance between the reaction term represented by $\partial f / \partial u$, the convection term $\partial f / \partial u_{x_{0}}$ and the diffusion coefficient $a_{00}(x)$.

Proposition 2.1. Let $S$ be a semilinear elliptic operator like in (2.1) with $f$ of class $\mathcal{C}^{1}$. If there exists an $\varepsilon>0$ such that

$$
\varepsilon^{2} a_{00}(x)+\varepsilon \frac{\partial f}{\partial p_{0}}(p, z, x)+\frac{\partial f}{\partial z}(p, z, x) \leq 0
$$


for all $(p, z, x) \in \mathbf{R}^{n+1} \times \mathbf{R} \times \bar{\Omega}$ (where $p=\left(p_{0}, p_{1}, \ldots, p_{n}\right)$ ) then $S$ satisfies hypothesis $\mathrm{H} 2$ with $\mu(x)=\exp \left(-\varepsilon x_{0}\right)$.

Proof: For fixed functions $u(x)$ and $v(x)$ the difference $S[u]-S[v]$ can be expressed by using the intermediate value theorem as

$$
\begin{aligned}
S[u]-S[v]= & \sum_{i, j=0}^{n} a_{i j}(x)(u-v)_{x_{i} x_{j}}+ \\
& +\sum_{i=0}^{n} \frac{\partial f}{\partial p_{i}}(\xi(x), \eta(x), x)(u-v)_{x_{i}}+\frac{\partial f}{\partial z}(\xi(x), \eta(x), x)(u-v) .
\end{aligned}
$$

Considering now the function $w=e^{-\varepsilon x_{0}}(u-v)$ we see that

$$
\begin{gathered}
S[u]-S[v]=e^{\varepsilon x_{0}}\left[\sum_{i, j=0}^{n} a_{i j}(x) w_{x_{i} x_{j}}+\right. \\
\left(\varepsilon^{2} a_{00}(x)+\varepsilon \frac{\partial f}{\partial p_{0}}(\xi, \eta, x)+\frac{\partial f}{\partial z}(\xi, \eta, x)\right) w+ \\
+ \text { linear terms in the first order derivatives of } w]
\end{gathered}
$$

So $S[u]-S[v]=e^{\varepsilon x_{0}} L[w]$, where $L$ is a linear elliptic operator for which the classical maximum and minimum principles hold, because of the sign condition (2.6), at least in bounded domains (see [7] Theorem 3.5).

But since $u$ and $v$ are bounded, and $\varepsilon>0$, in the case of $\Omega_{0}$ being unbounded it is clear that $w$ tends to zero uniformly on $\left(x_{1}, x_{2}, \ldots, x_{n}\right)$ when $x_{0}$ tends to infinity. So (2.3) and (2.4) also hold if $\Omega_{0}$ is unbounded. Indeed, let us assume, for instance, $S[u]-S[v] \geq 0$ (and so $L[w] \geq 0$ ) in $\Omega_{0}$ and that there exists $\bar{x}=\left(\bar{x}_{0}, \bar{x}_{1}, \ldots, \bar{x}_{n}\right) \in \Omega_{0}$ such that

$$
w(\bar{x}) \geq \max \left\{0, \sup _{\partial \Omega_{0}} w(x)\right\} .
$$

Then, there exists $M>\bar{x}_{0}$ such that $w(x) \leq \frac{1}{2} w(\bar{x})$ if $x_{0} \geq M$. Let us consider now the bounded domain $\widetilde{\Omega}_{0}=\Omega_{0} \cap\left\{x_{0}<M\right\}$, which contains $\bar{x}$. We have

$$
w(\bar{x}) \geq \max \left\{0, \sup _{\partial \Omega_{0} \cup\left\{x_{0}=M\right\}} w(x)\right\} \max \left\{0, \sup _{\partial \widetilde{\Omega}_{0}} w(x)\right\}
$$

and therefore $w$ is a constant in the connected component of $\widetilde{\Omega}_{0}$ that contains $\bar{x}$ due to the maximum principle (see [7] Theorem 3.5). As $M$ can be taken arbitrarily large and $\Omega_{0}$ is a domain, $w$ is a constant in $\Omega_{0}$. 
Remark: There is a trick to relax conditions like (2.6) in some situations. For example, in the particular case $a_{i j}(x) \equiv \delta_{i j}$, for $i \neq 0$ or $j \neq 0$, (related to the classical Laplacian case) and $f$ independent of $p,(2.6)$ can be weakened to

$$
\varepsilon^{2} a_{00}(x)+\frac{\partial f}{\partial z}(z, x) \leq-\lambda
$$

taking $\lambda$ any number strictly greater than $\lambda_{1}$, where $\lambda_{1}\left(\lambda_{1}<0\right)$ is the first eigenvalue of the Laplacian operator with Dirichlet boundary conditions in $\Omega^{\prime}$. This can be done by taking $\mu(x)=e^{-\varepsilon x_{0}} / \Lambda\left(x_{1}, \ldots, x_{n}\right)$ where $\Lambda\left(x_{1}, \ldots, x_{n}\right)$ is the first eigenfunction of the Laplacian operator with Dirichlet boundary conditions in a slightly larger domain $\Omega^{\prime \prime} \supset \supset \Omega^{\prime}$.

The following proposition, very easy to prove, shows a simple situation where (2.6) holds.

Proposition 2.2. Suppose that the following quantities are finite

$$
\begin{aligned}
A & =\sup \left\{a_{00}(x): x \in \bar{\Omega}\right\}, \\
B_{0} & =\sup \left\{\frac{\partial f}{\partial p_{0}}(p, z, x):(p, z, x) \in \mathbf{R}^{n+1} \times \mathbf{R} \times \bar{\Omega}\right\}, \\
C & =\sup \left\{\frac{\partial f}{\partial z}(p, z, x):(p, z, x) \in \mathbf{R}^{n+1} \times \mathbf{R} \times \bar{\Omega}\right\},
\end{aligned}
$$

and suppose also that $B_{0}^{2}-4 A C \geq 0$ and $-B_{0}+\sqrt{B_{0}^{2}-4 A C}>0$. Then (2.6) holds, at least for $\varepsilon=\left(-B_{0}+\sqrt{B_{0}^{2}-4 A C}\right) / 2 A$ (note that $A>0$, because of ellipticity).

Example. As an application of Proposition 2.2 above let us consider the semilinear operator

$$
S[u]=\Delta u+B_{0} u_{x_{0}}+C u-u^{3}
$$

and observe that if $4 C \leq B_{0}^{2}$ and $B_{0}<\sqrt{B_{0}^{2}-4 C}$, it satisfies hypothesis H2. So $C$ can be negative and $B_{0}$ arbitrary, but also $C$ can be taken non-negative provided that $B_{0}$ is large and negative.

Let us recall that $S$ is the semilinear operator defined by

$$
S[u]=\sum_{i, j=0}^{n} a_{i j}(x) u_{x_{i} x_{j}}+f(\nabla u, u, x) .
$$

Besides the hypotheses $\mathrm{H} 1$ and $\mathrm{H} 2$ we assume that $f(p, z, x)$ satisfies also the following

H3. $|f|=O\left(|p|^{2}\right)$ as $|p| \rightarrow \infty$ uniformly for $x \in \Omega$ and bounded $z$.

H4. $z f(0, z, x) \leq 0$ for $|z| \geq M_{0}$ and some $M_{0}>0$.

We have the following result of existence, uniqueness and continuous dependence 
Theorem 2.3. Let $\Omega$ and the operator $S$ satisfy H1-H4. Let us write $(s, y)$ instead of $\left(x_{0}, x_{1}, \ldots, x_{n}\right)$, so $s=x_{0}$ and $y=\left(x_{1}, x_{2}, \ldots, x_{n}\right)$. We claim that

i) The Dirichlet problem

$$
\begin{array}{ll}
S[u]=0 & \text { in } \Omega, \\
u\left(s_{0}, y\right)=\varphi(y) & \text { for } y \in \Omega^{\prime}, \\
u(s, y)=0 & \text { for } y \in \partial \Omega^{\prime}, \\
|u(s, y)| \text { bounded in } \Omega,
\end{array}
$$

has one and only one classical $\left(u \in \mathcal{C}(\bar{\Omega}) \cap \mathcal{C}^{2}(\Omega)\right)$ solution for any initial condition $\varphi \in \mathcal{C}_{0}^{0}\left(\overline{\Omega^{\prime}}\right)$.

ii) The solution $u$ depends on the initial condition $\varphi$ in a continuous way in the uniform norm for $\varphi$ and the topology of the uniform convergence on compact sets of $\bar{\Omega}$ for $u$.

Proof: Let us consider the auxiliary domains $Q_{k}=\left(s_{0}, s_{0}+k\right) \times \Omega^{\prime}, k=$ $1,2,3, \ldots$ and the following Dirichlet problems

$$
\begin{array}{ll}
S\left[u_{k}\right]=0 & \text { in } \quad Q_{k}, \\
u_{k}(s, y)=\varphi(y) & \text { for } s=s_{0} \text { or } s=s_{0}+k \text { and } y \in \overline{\Omega^{\prime}} \\
u_{k}(s, y)=0 & \text { for } \quad y \in \partial \Omega^{\prime} .
\end{array}
$$

Under our hypotheses these problems have at least one classical solution $u_{k} \in$ $\mathcal{C}\left(\overline{Q_{k}}\right) \cap \mathcal{C}^{2}\left(Q_{k}\right)$ (see [10] Theorem 8.3 of Chapter IV (p. 276) with the observations contained in the proofs of Theorem 8.7 of the same chapter (p. 285) and specially of Theorem 3.3 of Chapter VI (p. 342) both dealing with the case of non $\mathcal{C}^{2+\gamma}$ data, or, alternatively, see [7] Theorem 14.17 (p. 326)).

Let us see that the $u_{k}$ are uniformly bounded. Let

$$
M=\max \left\{M_{0}, \sup _{\Omega^{\prime}}|\varphi(y)|\right\} .
$$

Then, we can use (2.3) and (2.4) to compare $u_{k}$ with the functions $v^{+}(x) \equiv M$ and $v^{-}(x) \equiv-M$ because we deduce from condition (2.8) that $S\left[v^{+}\right] \leq 0$ and $S\left[v^{-}\right] \geq 0$. So we conclude that

$$
-M \leq u_{k}(x) \leq M
$$

for all $x \in Q_{k}$.

Let us now prove that the sequence $u_{\ell}$ is uniformly convergent in compact subsets of $\bar{\Omega}$, that is the sequence $u_{\ell}$ is uniformly convergent in each $Q_{k}$.

Let $\ell, m \geq k$ and, for definiteness, $\ell \leq m$. Since $S\left[u_{\ell}\right]-S\left[u_{m}\right]=0$ on $Q_{\ell} \supset Q_{k}$, by (2.5) we will have

$$
\begin{aligned}
\sup _{x \in Q_{k}}\left|\mu(x)\left(u_{\ell}(x)-u_{m}(x)\right)\right| & \leq \sup _{x \in Q_{\ell}}\left|\mu(x)\left(u_{\ell}(x)-u_{m}(x)\right)\right| \leq \\
& \leq \sup _{x \in \partial Q_{\ell}}\left|\mu(x)\left(u_{\ell}(x)-u_{m}(x)\right)\right| .
\end{aligned}
$$


By using that $\left|u_{m}(x)\right| \leq M$ we conclude that

$$
\sup _{x \in Q_{k}}\left|\mu(x)\left(u_{\ell}(x)-u_{m}(x)\right)\right| \leq 2 M \sup _{s=s_{0}+\ell}|\mu(x)|
$$

which can also be written as

$$
\left\|\mu\left(u_{\ell}-u_{m}\right)\right\|_{\mathcal{C}^{\circ}\left(Q_{k}\right)} \leq \underset{s-s_{0}=\min (m, \ell)}{2 M} \mid
$$

and this tends to zero as $m, \ell \rightarrow \infty$.

With this we have seen the existence of a limit function $u \in \mathcal{C}^{0}(\bar{\Omega})$, bounded by $M$, that satisfies the boundary conditions. Let us see that $u \in \mathcal{C}^{2}(\Omega)$ and satisfies the equation.

Let $B$ be any open ball $B \subset \subset \Omega$. Theorem 3.1 of Chapter IV of [10] (p. 244) (or Theorem 14.6 of $[7]$ (p. 320)) shows that

$$
\left\|u_{k}\right\|_{\mathcal{C}^{1}(\bar{B})} \leq C
$$

where $C$ does not depend on $k$. Then, an important result of Ladyženskaja and Ural'ceva implies that there exist $\alpha>0$ and $C_{1}>0$, independent of $k$, such that

$$
\left\|u_{k}\right\|_{\mathcal{C}^{1+\alpha}\left(\overline{B_{1}}\right)} \leq C_{1}
$$

(see [10] Theorem 6.1 of Chapter IV (p. 258) or [7], Theorem 12.1 (p. 265)) where $B_{1}$ is a smaller concentric ball.

Now the equation $S\left[u_{k}\right]=0$ in $B_{1}$ can be seen as a linear equation for $u_{k}$ with a $\mathcal{C}^{\alpha}$ right-hand side $f$. And by Schauder interior estimates ([10], inequality 1.12 of Chapter III (p. 203), or [7] Theorem 6.2 on p. 85) we conclude that

$$
\left\|u_{k}\right\|_{\mathcal{C}^{2+\alpha}\left(\overline{B_{2}}\right)} \leq C_{2}
$$

where $B_{2}$ is an even smaller concentric ball and $C_{2}$ is also independent of $k$.

By a compactness argument, since $u_{k}$ tends to $u$ in $\mathcal{C}^{0}\left(\overline{B_{2}}\right)$ it holds that $u_{k}$ tends to $u$ in $\mathcal{C}^{2+\beta}\left(\overline{B_{2}}\right)$ with $\beta<\alpha$, and taking limits in the expression $S\left[u_{k}\right]=0$ we see that $S[u]=0$ in $\overline{B_{2}}$ too. Since $\overline{B_{2}}$ is a neighbourhood of an arbitrary point in $\Omega$, we conclude that $u \in \mathcal{C}^{2}(\Omega)$ and satisfies the equation everywhere in $\Omega$.

This proves existence. To prove uniqueness, let $u$ and $v$ be bounded functions in $\mathcal{C}^{0}(\bar{\Omega}) \cap \mathcal{C}^{2}(\Omega)$ such that $u(s, y)=v(s, y)=0$ for $y \in \partial \Omega^{\prime}$ and $S[u]=S[v]=0$. The inequality (2.5) with $\Omega_{0}=\Omega$ gives us

$$
\sup _{x \in \Omega}|\mu(x)(u(x)-v(x))| \leq\left\|\mu\left(s=s_{0}\right)\left(u\left(s=s_{0}\right)-v\left(s=s_{0}\right)\right)\right\|_{\mathcal{C}^{\circ}\left(\overline{\Omega^{\prime}}\right)} .
$$

This inequality proves both uniqueness and continuity with respect to the initial value. This finishes the proof of i) and ii).

The following result shows the regularizing feature of the problem 
Theorem 2.4. Let $\Omega$ and the operator $S$ satisfy H1-H4. Let $\Omega_{k}:=\left(s_{0}+1 / k, s_{0}+\right.$ $k) \times \Omega^{\prime}, k=2,3, \ldots$. We claim that the solutions $u$ of $(2.9)$ belong to $\mathcal{C}^{2+\gamma}\left(\overline{\Omega_{k}}\right)$ for all $k$ and if $\|\varphi\|_{\mathcal{C}^{\circ}\left(\overline{\Omega^{\prime}}\right)} \leq R$ then $\|u\|_{\mathcal{C}^{2+\gamma}\left(\overline{\Omega_{k}}\right)} \leq C$, where $C$ depends on $R$ (and, of course, on $k, \Omega^{\prime}$ and the functions defining $S$ ) but otherwise not on $\varphi$.

Before going into the proof let us start with a lemma that contains partially interior a priori bounds for the solutions of $S[u]=0$. We will tell the reader how to follow the path of the proof along the references [10] and [7].

Lemma 2.5. Under the hypotheses of the theorem above, and using the same notation we claim that if $v \in \mathcal{C}^{2+\gamma}\left(\overline{\Omega_{k+1}}\right)$ is a solution of $S[v]=0$ in $\Omega_{k+1}$ with $v(s, y)=0$ for $y \in \partial \Omega^{\prime}$ such that $\|v\|_{\mathcal{C}^{\circ}\left(\overline{\Omega_{k+1}}\right)} \leq R$ then $\|v\|_{\mathcal{C}^{2+\gamma}\left(\overline{\Omega_{k}}\right)} \leq C$, where $C$ depends on $R$ (and on $k, \Omega^{\prime}$ and $S$ ) but otherwise not on $v$.

Proof: According to the proofs of [10] Lemma 2.1 of Chapter VI (p. 322) (or [7] Theorem 13.1 on p. 282) it is clear that under our hypotheses, the boundary estimate

$$
|\nabla v(s, y)| \leq C_{1} \quad \text { for } \quad(s, y) \in\left[s_{0}+\frac{1}{k+1}, s_{0}+k+1\right] \times \partial \Omega^{\prime}
$$

holds, where $C_{1}$ depends on $R$, but otherwise not on $v$. Note that the mentioned results deal with estimates of $|\nabla v|$ on the whole boundary of a domain, and not on a part of it, but an inspection of the proofs gives us so.

Let us now use the self-explained notation $\Omega_{r}$ for non-integer values of $r$. At this point we can apply Lemma 4.2 of Chapter IV of [10] (p. 248) (see also the proof of Theorem 14.7 of $[\mathbf{7}]$ on p. 320) and we deduce that

$$
|\nabla v(s, y)| \leq C_{2} \quad \text { for } \quad(s, y) \in \overline{\Omega_{k+3 / 5}}
$$

where $C_{2}$ depends on $C_{1}$ and $R$ and on the value of

$$
\int_{\Omega_{k+4 / 5}} \sum_{i, j=0}^{n} v_{x_{i} x_{j}}^{2} d x
$$

but otherwise not on $v$. And in the proof of Theorem 5.1 of the same chapter of [10] (p. 254) it is shown how to bound the value of this integral in terms of $R$.

An important result of Ladyženskaja and Ural'ceva is now that the $\mathcal{C}^{1}$ bounds imply $\mathcal{C}^{1+\alpha}$ bounds. This is Theorem 6.5 of Chapter IV of $[\mathbf{1 0}]$ (p. 261) where it is stated as a global estimate, but it can be stated as a partially interior estimate as in a remark after Theorem 12.7 of [7] (p. 276). In any case, there exist $\alpha_{k}$ and $C_{3}$ depending on $v$ only through $R$ such that

$$
\|v\|_{\mathcal{C}^{1+\alpha_{k}\left(\overline{\Omega_{k+2 / 5}}\right.} \leq} \leq C_{3} .
$$

Now the equation $S[v]=0$ can be seen as a linear elliptic equation for $v$ in $\overline{\Omega_{k+2 / 5}}$ with a right hand side whose $\mathcal{C}^{\alpha_{k}}$ norm is bounded in terms of $R$. Aplying 
Schauder partially-interior estimates ([7] Corollary 6.7 on p. 95 or $[\mathbf{1 0}]$ inequality 1.13 of Chapter III p. 103) we see that

$$
\|v\|_{\mathcal{C}^{2+\alpha_{k}}\left(\overline{\Omega_{k+1 / 5}}\right)} \leq C_{4}
$$

where $C_{4}$ depends on $v$ only through $R$. In prevision of the possibility of having $\alpha_{k}<\gamma$ there is still place to repeat the partially-interior estimate of Schauder and obtain

$$
\|v\|_{\mathcal{C}^{2+\gamma}\left(\overline{\Omega_{k}}\right)} \leq C
$$

as we were willing to prove.

Proof of Theorem 2.4: To prove Theorem 2.4 we re-start again the proof of Theorem 2.3, in order to improve the regularity. Let us consider, to begin, that $\|\varphi\| \leq R, \varphi \in \mathcal{C}_{0}^{2+\gamma}\left(\overline{\Omega^{\prime}}\right)$. Let $D_{\ell}$ be a collection of smooth $\left(\mathcal{C}^{2+\gamma}\right)$ domains such that

$$
\Omega_{\ell} \subset D_{\ell} \subset \Omega_{\ell+1}
$$

and let us consider the auxiliary problems

$$
\begin{aligned}
& S\left[v_{\ell}\right]=0 \quad \text { in } D_{\ell}, \\
& v_{\ell}(s, y)=\varphi(y) \text { in } \partial D_{\ell} \text {, }
\end{aligned}
$$

that have, at least, one solution $v_{\ell}$ which is smooth up to the boundary: $v_{\ell} \in$ $\mathcal{C}^{2+\gamma}\left(\overline{D_{\ell}}\right)$ (see $[\mathbf{1 0}]$ Theorem 8.3 of Chapter IV (p. 276) or [7] Theorem 14.9 (p. $322)$ ). By the same argument we used in the beginning of the proof of Theorem 2.3 for the $u_{k}$ it is clear that

$$
\left|v_{\ell}(x)\right| \leq M=\max \left\{M_{0}, R\right\}
$$

Let us denote also by $v_{\ell}(x)$ the extension to all $\Omega$ of $v_{\ell}$ defined by $v_{\ell}(s, y)=\varphi(y)$ if $(s, y) \notin D_{\ell}$. The functions $v_{\ell}$ are now continuous in $\bar{\Omega}$ and still bounded by the same $M$.

Let us show that this sequence $v_{\ell}$ approaches the solution $u$ obtained in Theorem 2.3 in the uniform sense on each of the compact sets $\overline{Q_{k}}=\left[s_{0}, s_{0}+k\right] \times \overline{\Omega^{\prime}}$. We have

$$
\begin{aligned}
\left\|\mu\left(u-v_{\ell}\right)\right\|_{\mathcal{C}^{\circ}\left(\overline{Q_{k}}\right)} & =\max \left(\left\|\mu\left(u-v_{\ell}\right)\right\|_{\mathcal{C}^{\circ}\left(\overline{Q_{k}}-D_{\ell}\right)},\left\|\mu\left(u-v_{\ell}\right)\right\|_{\mathcal{C}^{\circ}\left(\overline{D_{\ell}} \cap \overline{Q_{k}}\right)}\right) \leq \\
& \leq \max \left(\|\mu(u-\varphi)\|_{\mathcal{C}^{\circ}\left(\overline{Q_{k}}-D_{\ell}\right)}, \sup _{x \in \partial \overline{D_{\ell}}}\left|\mu(x)\left(u(x)-v_{\ell}(x)\right)\right|\right) .
\end{aligned}
$$

The first quantity tends to zero as $\ell \rightarrow \infty$ since $u$ is uniformly continuous in a neighbourhood of $s=s_{0}$ and $u\left(s_{0}, y\right)=\varphi(y)$. For the second quantity we distinguish three parts of $\partial \overline{D_{\ell}}$. The front boundary: $\partial \overline{D_{\ell}} \cap\left\{s_{0}<s<s_{0}+1 / \ell\right\}$. This part needs not to be taken into account since it is already considered in the quantity $\|\mu(u-\varphi)\|_{\mathcal{C}^{\circ}\left(\overline{Q_{k}}-D_{\ell}\right)}$. The lateral boundary: $\partial \overline{D_{\ell}} \cap\left\{s_{0}+1 / \ell \leq s \leq\right.$ 
$\left.s_{0}+\ell\right\}$. On this part $u(x)=v_{\ell}(x)=0$. The back boundary: $\partial \overline{D_{\ell}} \cap\left\{s_{0}+\ell<s\right\}$. On this part it is clear that $|\mu(x)|$ tends to zero as $\ell \rightarrow \infty$. So $v_{\ell}$ tends to $u$ uniformly on compact sets.

As a consequence of the previous Lemma 2.5, if $\ell>k$ then

$$
\left\|v_{\ell}\right\|_{\mathcal{C}^{2+\gamma}\left(\overline{\Omega_{k}}\right)} \leq C
$$

where $C$ depends on $R$ but not on $\ell$. As this is a closed property with respect to the uniform convergence on $\Omega_{k}$, we deduce that $u \in \mathcal{C}^{2+\gamma}\left(\overline{\Omega_{k}}\right)$ and also that

$$
\|u\|_{\mathcal{C}^{2+\gamma}\left(\overline{\Omega_{k}}\right)} \leq C
$$

with the same constant $C$ as before. Now, if our initial condition $\varphi \in \mathcal{C}_{0}^{0}\left(\overline{\Omega^{\prime}}\right)$ does not belong to $\mathcal{C}^{2+\gamma}\left(\overline{\Omega^{\prime}}\right)$, we can aproximate it by a sequence $\varphi_{m}$ of functions of $\mathcal{C}_{0}^{2+\gamma}\left(\overline{\Omega^{\prime}}\right)$, the approximation being in the $\mathcal{C}^{0}\left(\overline{\Omega^{\prime}}\right)$-norm. Then the corresponding solutions $u_{m}(x)$ will converge to $u$ in $\mathcal{C}^{0}\left(\overline{\Omega_{k}}\right)$, according to $(2.10)$. So we will still have $u \in \mathcal{C}^{2+\gamma}\left(\overline{\Omega_{k}}\right)$ and

$$
\|u\|_{\mathcal{C}^{2+\gamma}\left(\overline{\Omega_{k}}\right)} \leq C
$$

with the same constant $C$ as before. This proves Theorem 2.4.

The following proposition will be of use in Section 4:

Proposition 2.6. Let $\Omega$ and the operator $S$ satisfy the hypotheses $\mathrm{H} 1-\mathrm{H} 4$ and further that

$$
\nu_{1} \sum_{i=0}^{n} \xi_{i}^{2} \leq \sum_{i, j=0}^{n} a_{i j}(x) \xi_{i} \xi_{j} \leq \nu_{2} \sum_{i=0}^{n} \xi_{i}^{2}
$$

for some $\nu_{1}, \nu_{2}>0$ and all $x \in \bar{\Omega}$ and $\left(\xi_{0}, \xi_{1}, \ldots, \xi_{n}\right) \in \mathbf{R}^{n+1}$, and also that

$$
\left|\frac{\partial a_{i j}(x)}{\partial x_{k}}\right| \leq \nu_{3}
$$

for some $\nu_{3}>0$ and all $x \in \bar{\Omega}$ and $i, j, k=0,1, \ldots, n$.

Let $v \in \mathcal{C}^{0}(\bar{\Omega}) \cap \mathcal{C}^{2}(\Omega)$ be a solution of $S[v]=0$ in $\Omega$ with $v(s, y)=0$ for $y \in \partial \Omega^{\prime}$ and $v \in \mathcal{C}^{2+\gamma}\left(\overline{\Omega_{k}}\right)$ for all $k$, with $\Omega_{k}=\left(s_{0}+\frac{1}{k}, s_{0}+k\right) \times \Omega^{\prime}$. Then, given $\delta>0$ and $R>0$ there exists a $C>0$, depending on $R$ and $\delta$ but otherwise not on $v$, such that if $\|v\|_{\mathcal{C}^{\circ}(\bar{\Omega})} \leq R$ then $\|\nabla v\|_{\mathcal{C}^{\circ}\left(\left[s_{0}+\delta, \infty\right) \times \overline{\Omega^{\prime}}\right)} \leq C$.

Remark: Note that (2.11) and (2.12) follow from hypothesis H1 in the two important cases where the functions $a_{i j}(x)$ and $f(p, z, x)$ do not depend on $x_{0}$ or are periodic in $x_{0}$.

Proof: Let us write hypothesis H3 in the form

$$
|f(p, z, x)| \leq c(|z|)\left(1+|p|^{2}\right)
$$


for some non-decreasing function $c$.

A careful analysis of the first two paragraphs of the proof of Lemma 2.5 shows that for our solution $v$ the quantity $\|\nabla v\|_{\mathcal{c}^{\circ}\left(\Omega_{k}\right)}$ can be bounded in terms of $R$, $\nu_{1}, \nu_{2}, \nu_{3}, c(R)$ and the geometry of $\Omega_{k}$ and $\Omega_{k+1}$. But the geometry and the other parameters remain unchanged if we replace $s_{0}$ by $s_{1} \geq s_{0}$. This proves our claim taking $k>\frac{1}{\delta}$ and observing that

$$
\|\nabla v\|_{\mathcal{C}^{\circ}\left(\left[s_{0}+\delta, \infty\right) \times \overline{\Omega^{\prime}}\right)} \leq \sup _{s_{1} \geq s_{0}}\|\nabla v\|_{\mathcal{C}^{\circ}\left(\left[s_{1}+\frac{1}{k}, s_{1}+k\right] \times \overline{\Omega^{\prime}}\right)}
$$

The following result shows that the problem is strongly order-preserving.

Theorem 2.7. Suppose that $S$ satisfies hypotheses $\mathrm{H} 1$ and $\mathrm{H} 2$ and that $u, v \in$ $\mathcal{C}^{0}(\bar{\Omega}) \cap \mathcal{C}^{2}(\Omega)$ satisfy

$$
\begin{array}{ll}
S[u]=S[v]=0 & \text { in } \Omega, \\
u\left(s_{0}, y\right) \geq v\left(s_{0}, y\right) \quad & \text { for } y \in \Omega^{\prime}, \\
u(s, y)=v(s, y)=0 & \text { for } y \in \partial \Omega^{\prime}, \\
|u(s, y)| \text { and }|v(s, y)| & \text { bounded in } \Omega .
\end{array}
$$

Then $u(s, y)>v(s, y)$ for all $(s, y) \in \Omega$ unless $u \equiv v$.

Proof: This is just the strong maximum principle. We see that $S[u]-S[v]=$ $0 \leq 0$ in $\Omega$. Then, because of $(2.4)$ we obtain that

$$
\mu(x)(u(x)-v(x))>\min \left\{0, \inf _{y \in \Omega^{\prime}} \mu\left(s_{0}, y\right)\left(u\left(s_{0}, y\right)-v\left(s_{0}, y\right)\right)\right\}=0
$$

for all $x \in \Omega$ unless $\mu(u-v)$ is a constant. So $u(x)>v(x)$ unless $\mu(u-v)$ is a constant. But if it is a constant it must be zero because it vanishes for $y \in \partial \Omega^{\prime}$.

\section{Autonomous and periodic equations}

Along this Section we will assume the hypotheses on $S$ and $\Omega^{\prime}$ of Theorem 2.3 and, furthermore, that the functions $a_{i j}(x)$ and $f(p, z, x)$ do not depend on $x_{0}$ (autonomous case) or, alternatively, that there exists $\omega>0$ (which will be assumed to be equal to $2 \pi$ without loss of generality) such that

$$
a_{i j}\left(x_{0}+\omega, x_{1}, \ldots, x_{n}\right)=a_{i j}\left(x_{0}, x_{1}, \ldots, x_{n}\right)
$$

and

$$
f\left(p, z, x_{0}+\omega, x_{1}, \ldots, x_{n}\right)=f\left(p, z, x_{0}, x_{1}, \ldots, x_{n}\right)
$$


for all $p, z, x$ (periodic case). Observe that in both cases the coefficient functions $a_{i j}$ are of class $\mathcal{C}^{1+\gamma}$ on the whole $\mathbf{R} \times \overline{\Omega^{\prime}}$ and also that $f$ is of class $\mathcal{C}^{1}$ on $\mathbf{R}^{n+1} \times \mathbf{R} \times \mathbf{R} \times \overline{\Omega^{\prime}}$.

We will prove in both cases the existence of a compact global attractor of the solutions, roughly a compact subset of $\mathcal{C}_{0}^{0}\left(\overline{\Omega^{\prime}}\right)$ to which the solutions of problem (2.9) tend when $s$ tends to infinity, uniformly for $\varphi$ taken in bounded subsets of $\mathcal{C}_{0}^{0}\left(\overline{\Omega^{\prime}}\right)$.

In the autonomous case, this will imply the existence of at least one "equilibrium solution", i.e., a function $v(y)$ belonging to $\mathcal{C}_{0}^{0}\left(\overline{\Omega^{\prime}}\right) \cap \mathcal{C}^{2}\left(\Omega^{\prime}\right)$ such that $u(s, y):=$ $v(y)$ satisfies (2.9) with $\varphi(y)=v(y)$. And in the periodic case, this will prove the existence of at least one "periodic solution", i.e., a function $u(s, y)$ belonging to $\mathcal{C}^{0}(\bar{\Omega}) \cap \mathcal{C}^{2}(\Omega)$ and satisfying

$$
\begin{array}{ll}
S[u]=0 & \text { in } \quad \Omega, \\
u(s, y)=0 & \text { for } \quad y \in \partial \Omega^{\prime}, \\
u(s+\omega, y)=u(s, y) & \text { for } \quad(s, y) \in \Omega .
\end{array}
$$

Of course, the existence of these particular solutions could be obtained also by others methods, without appeal to compact attractors.

Along this section we will make use of the theory of dissipative infinite dimensional dynamical systems developed in [8].

Let us begin by stating the following two propositions which can be obtained from Theorem 2.3 and Theorem 2.4.

Proposition 3.1. In the autonomous case, let $u(s, y)$ be the only (classical) solution of the Dirichlet problem

$$
\begin{array}{ll}
S[u]=0 & \text { in } \quad \Omega=(0, \infty) \times \Omega^{\prime}, \\
u(0, y)=\varphi(y) \quad \text { for } \quad y \in \Omega^{\prime}, \\
u(s, y)=0 \quad \text { for } \quad y \in \partial \Omega^{\prime}, \\
|u(s, y)| \text { bounded in } \Omega .
\end{array}
$$

Then $T(s) \varphi:=u(s, \cdot)$ is a (nonlinear) $\mathcal{C}^{0}$-semigroup in the Banach space $X:=$ $\mathcal{C}_{0}^{0}\left(\overline{\Omega^{\prime}}\right)$ which is completely continuous for $s>0$.

Proof: As usual, the semigroup property follows from the uniqueness of solution of problem (2.9) (part i) of Theorem 2.3).

Now, let $u_{\varphi}$ and $u_{\psi}$ be the solutions of (3.1) corresponding to initial conditions $\varphi$ and $\psi$ respectively. Then

$$
\left|u_{\varphi}(s, y)-u_{\psi}\left(s^{\prime}, y\right)\right| \leq\left|u_{\varphi}(s, y)-u_{\varphi}\left(s^{\prime}, y\right)\right|+\left|u_{\varphi}\left(s^{\prime}, y\right)-u_{\psi}\left(s^{\prime}, y\right)\right| .
$$

The first term on the right hand side is small, uniformly for $y \in \overline{\Omega^{\prime}}$, if $\left|s-s^{\prime}\right|$ is sufficiently small because $u_{\varphi}$ is uniformly continuous in $[s-\delta, s+\delta] \times \overline{\Omega^{\prime}}$ due to part i) of Theorem 2.3. The second term is also small, uniformly for $y \in \overline{\Omega^{\prime}}$, 
provided that $\sup \left\{|\varphi(y)-\psi(y)|: y \in \Omega^{\prime}\right\}$ is small and $s^{\prime} \in[s-\delta, s+\delta]$ due to part ii) of Theorem 2.3.

This gives the joint continuity of $T(s) \varphi$ with respect to $(s, \varphi) \in[0, \infty) \times X$. Given $s>0$ and a bounded subset $B$ of $X$, the precompactness of $T(s) B$ in $X$ follows immediately from Theorem 2.4 because it ensures that $T(s) B$ is a bounded subset of $\mathcal{C}^{2+\gamma}\left(\overline{\Omega^{\prime}}\right)$, and this space is compactly embedded in $X$.

Similarly, we obtain the following

Proposition 3.2. In the periodic case (with $\omega=2 \pi$ ), let $u^{s_{0}}(s, y)$ be the only solution of problem (2.9). Then $\widetilde{T}(s)\left(s_{0}, \varphi\right):=\left(\left(s_{0}+s\right)_{\bmod 2 \pi}, u^{s_{0}}\left(s_{0}+s, \cdot\right)\right)$ is a (nonlinear) $\mathcal{C}^{0}$-semigroup in $Y:=S^{1} \times X$ (with $X:=\mathcal{C}_{0}^{0}\left(\overline{\Omega^{\prime}}\right)$ ) which is completely continuous for $s>0$.

On the other hand, $T \varphi:=u^{0}(2 \pi, \cdot)$ is a continuous map in $X$ which is completely continuous.

Let us now state two propositions in the setting of abstract theory of dynamical systems. They give a condition of dissipativeness without a Lyapunov function but with an idea based on the maximum principle. They will be used in the proofs of Theorems 3.5 and 3.6 below.

Proposition 3.3. a) Let $T$ be a continuous map in a complete metric space $X$ satisfying the following hypotheses

i) The set $\left\{T^{k} x: k \geq 0\right\}$ is precompact for all $x \in X$.

ii) There exists a continuous function $V: X \rightarrow[0, \infty)$ with the level sets bounded and a positive number $M_{0}$ such that if $V\left(T^{j} x\right) \geq V\left(T^{k} x\right)$ for some $j>0$ and any $k \geq 0$ then $V\left(T^{j} x\right) \leq M_{0}$.

Then the bounded set $B=\left\{x \in X: V(x) \leq M_{0}\right\}$ attracts every point of $X$ under the discrete semigroup generated by $T$.

b) Let $T(s)$ a $\mathcal{C}^{0}$-semigroup in $X$ satisfying the same hypotheses as $T$ above, substituting the integer numbers $j$ and $k$ by real numbers $\bar{s}>0$ and $s \geq 0$ respectively. Then the set $B$ attracts every $x \in X$ under the semigroup $T(s)$.

Proof: a) Let $x$ be any point in $X$. The $\omega$-limit set of $x, \omega(x)$, is non-empty, compact, invariant and attracts $x$ (see Lemma 2.1.2 [8]). We will show that $\omega(x) \subset B$.

Let $y \in \omega(x)$ be a point where $V$ attains its maximum value on $\omega(x)$ and let us take $z \in \omega(x)$ such that $T z=y$. The existence of $z$ follows from the invariance of the $\omega$-limit set. Then,

$$
V(y)=V(T z) \geq V\left(T^{k} z\right) \text { for all } k \geq 0
$$

again due to the invariance of $\omega(x)$. So, $V(y) \leq M_{0}$ by ii) and, hence, $\omega(x) \subset B$. The statement follows because $\omega(x)$ attracts $x$. 
b) The proof of part b) is analogous, taking $z \in \omega(x)$ such that $T(1) z=y(\omega(x)$ is non-empty, compact, invariant and attracts $x$, see Lemma 3.1.2 [8]). Then,

$$
V(y)=V(T(1) z) \geq V(T(s) z) \text { for all } s \geq 0,
$$

and the statement follows.

Proposition 3.4. a) Let $T$ be a continuous map in a complete metric space $X$, which is completely continuous, has the orbits (of points) bounded and satisfies hypothesis ii) of Proposition 3.3 above. Then there exists a global attractor (a maximal invariant compact set which attracts bounded sets of $X$ under $T$ ). Moreover, if $X$ is a Banach space, $T$ has a fixed point and the attractor is connected.

b) Let $T(s)$ be a $\mathcal{C}^{0}$-semigroup in $X$, which is completely continuous for $s>0$, has the orbits (of points) bounded, and satisfies ii) of Proposition 3.3 above substituting the integer numbers $j$ and $k$ by real numbers $\bar{s}>0$ and $s \geq 0$. Then $T(s)$ has a global attractor. If $X$ is a Banach space, the global attractor is connected and $T(s)$ has an equilibrium point.

Proof: The hypotheses of this proposition imply hypothesis i) of Proposition 3.3 in both cases. So, $T$ and $T(s)$ are point dissipative. The statement follows from Theorems 2.4.7 and 3.4.8 and Corollary 2.6.3 of [8].

Theorem 3.5. The semigroup $T(s)$ defined in Proposition 3.1 has a connected global attractor in $X=\mathcal{C}_{0}^{0}\left(\overline{\Omega^{\prime}}\right)$ and has also an equilibrium point.

Proof: Since the orbits of $T(s)$ are bounded by construction, we have only to prove that $T(s)$ satisfies the last hypothesis of Proposition $3.3 \mathrm{~b}$ ) and the statement will follow from Corollary $3.4 \mathrm{~b})$.

Let $\varphi \in X$, let $u(s, \cdot)=T(s) \varphi$ and let us assume that there exists $\bar{s}>0$ such that

$$
\sup _{y \in \Omega^{\prime}}|u(\bar{s}, y)|=\|T(\bar{s}) \varphi\| \geq\|T(s) \varphi\|=\sup _{y \in \Omega^{\prime}}|u(s, y)| \text { for all } s \geq 0 .
$$

Let $\bar{y} \in \overline{\Omega^{\prime}}$ be a point where the continuous function

$$
\overline{\Omega^{\prime}} \ni y \mapsto|u(\bar{s}, y)|
$$

attains its maximum value.

Notice that $\bar{y}$ can be chosen in $\Omega^{\prime}$ because otherwise the boundary condition implies $u(\bar{s}, y)=0$ for all $y \in \overline{\Omega^{\prime}}$.

So, $(\bar{s}, \bar{y}) \in \Omega$ is an interior maximum point (if $u(\bar{s}, \bar{y}) \geq 0$ ) or an interior minimum point (if $u(\bar{s}, \bar{y}) \leq 0$ ) of function $u$. In both cases, this implies that $|u(\bar{s}, \bar{y})| \leq M_{0}$ (and so, $\left.\|T(\bar{s}) \varphi\| \leq M_{0}\right)$ due to hypotheses $\mathrm{H} 2$ and $\mathrm{H} 4$. Indeed, 
assume for instance that $(\bar{s}, \bar{y})$ is an interior maximum point and that $u_{0}:=$ $u(\bar{s}, \bar{y})>M_{0}$, and define $v^{+}(s, y) \equiv u_{0}$. Then,

$$
S[u]-S\left[v^{+}\right]=-f\left(0, u_{0}, x\right) \geq 0,
$$

and by hypothesis H2, either $u(x)-v^{+}(x)<0$ in $\Omega$ or $u(x) \equiv v^{+}(x) \equiv u_{0}$ in $\Omega$. The first possibility leads to a contradiction: for all $x \in \Omega, u(x)<v^{+}(x)=u_{0}=$ $u(\bar{s}, \bar{y})$. And also the second possibility can not happen because of the boundary conditions, and $u_{0}$ being positive.

Therefore, the last hypothesis of Proposition $3.3 \mathrm{~b}$ ) holds by taking $V=\|\cdot\|$.

Theorem 3.6. With the definitions of Proposition 3.2 we have

a) The map $T$ has a connected global attractor $A$ in $X . T$ has also a fixed point.

b) The semigroup $\tilde{T}(s)$ has a global attractor $\tilde{A}$ in $Y$ and $\widetilde{A}=\{\widetilde{T}(s)(\{0\} \times A)$ : $0 \leq s<2 \pi\}$. Moreover, $\widetilde{T}(s)$ has a periodic trajectory of period $2 \pi$.

Proof: a) As above, we have only to show that $T$ satisfies hypothesis ii) of Proposition $3.3 \mathrm{a}$ ) and the claim will follow from Corollary $3.4 \mathrm{a}$ ).

Let $\varphi \in X$ and let $u^{0}(s, y)$ be the solution of problem (2.9) with $s_{0}=0$. Notice that $u^{0}(2 k \pi, \cdot)=T^{k} \varphi$.

Let us assume the existence of an integer $j>0$ such that

$$
\sup _{y \in \Omega^{\prime}}\left|u^{0}(2 j \pi, y)\right|=\left\|T^{j} \varphi\right\| \geq\left\|T^{k} \varphi\right\|=\sup _{y \in \Omega^{\prime}}\left|u^{0}(2 k \pi, y)\right|
$$

for all $k \geq 0$.

This implies that the maximum value of the function $\left|u^{0}(s, y)\right|$ on the compact set $R:=[2(j-1) \pi, 2(j+1) \pi] \times \overline{\Omega^{\prime}}$ is attained at an interior point $(\bar{s}, \bar{y}) \in \Omega$. As in the proof of Theorem 3.5, this implies $\left|u^{0}(\bar{s}, \bar{y})\right| \leq M_{0}$, and so,

$$
\left\|T^{j} \varphi\right\|=\sup _{y \in \Omega^{\prime}}\left|u^{0}(2 j \pi, y)\right| \leq \sup _{R}\left|u^{0}(s, y)\right|=\left|u^{0}(\bar{s}, \bar{y})\right| \leq M_{0} .
$$

Therefore, hypothesis ii) of Proposition 3.3 a) holds with $V=\|\cdot\|$.

b) The proof of the existence of the global attractor reduces again to show that the semigroup $\widetilde{T}(s)$ satisfies the last hypothesis of Proposition $3.3 \mathrm{~b}$ ). Let us define

$$
\begin{aligned}
V: Y & \longrightarrow[0, \infty) \\
(s, \varphi) & \longrightarrow\|\varphi\|
\end{aligned}
$$

$V$ is continuous and has the level sets bounded.

If there exist $\bar{s}>0$ and $\left(s_{0}, \varphi\right) \in Y$ such that

$$
\sup _{y \in \Omega^{\prime}}\left|u^{s_{0}}\left(s_{0}+\bar{s}, y\right)\right|=V\left(\widetilde{T}(\bar{s})\left(s_{0}, \varphi\right)\right) \geq V\left(\widetilde{T}(s)\left(s_{0}, \varphi\right)\right)=\sup _{y \in \Omega^{\prime}}\left|u^{s_{0}}\left(s_{0}+s, y\right)\right|
$$


for all $s \geq 0$, it follows that the function $\left|u^{s_{0}}(s, y)\right|$ has an interior maximum point $\left(s_{0}+\bar{s}, \bar{y}\right) \in \Omega=\left(s_{0}, \infty\right) \times \Omega^{\prime}$.

As in the proof of Theorem 3.5 we obtain $\left|u^{s_{0}}\left(s_{0}+\bar{s}, \bar{y}\right)\right| \leq M_{0}$ and, hence,

$$
V\left(\widetilde{T}(\bar{s})\left(s_{0}, \varphi\right)\right)=\sup _{y \in \Omega^{\prime}}\left|u^{s_{0}}\left(s_{0}+\bar{s}, y\right)\right|=\left|u^{s_{0}}\left(s_{0}+\bar{s}, \bar{y}\right)\right| \leq M_{0} .
$$

To prove the second statement of part b), let us notice that

$$
\widetilde{T}(2 \pi)(\{0\} \times A)=\{0\} \times T A=\{0\} \times A=\widetilde{T}(0)(\{0\} \times A)
$$

where we have used the invariance of $A$ under $T$. So, the set $\{\widetilde{T}(s)(\{0\} \times A)$ : $0 \leq s<2 \pi\}$ is compact, because it is the image of the compact set $[0,2 \pi] \times\{0\} \times A$ by a continuous map, and invariant. Therefore, it is contained in $\widetilde{A}$, which is the maximal compact invariant set.

On the other hand, let $\left(s_{0}, \varphi\right)$ be any point in $\tilde{A}$. As $\tilde{A}$ is invariant and compact, there is a complete orbit through $\left(s_{0}, \varphi\right)$ which is a precompact set in $Y$. So, the set $\left\{\psi \in X\right.$ : there exists $s \geq 0$ such that $\widetilde{T}(s)(0, \psi)=\left(s_{0}, \varphi\right)$ or $\left.\widetilde{T}(s)\left(s_{0}, \varphi\right)=(0, \psi)\right\}$ is a (non-empty) precompact and invariant (under $T$ ) subset of $X$. Therefore it must be contained in $A$. In particular, there is $\psi_{0} \in A$ such that $\left(s_{0}, \varphi\right)=\widetilde{T}\left(s_{0}\right)\left(0, \psi_{0}\right) \in\{\widetilde{T}(s)(\{0\} \times A), 0 \leq s<2 \pi\}$.

Finally, the last claim follows directly from the last statement of part a) because, if $T \varphi=\varphi$ then $\widetilde{T}(2 \pi)(0, \varphi)=\left(0, u^{0}(2 \pi, \cdot)\right)=(0, T \varphi)=(0, \varphi)$.

\section{Convergence to zero}

In this section we give conditions on the nonlinear function $f(p, z, x)$ ensuring that the solutions $u(s, y)$ tend to zero as $s$ tends to infinity, uniformly on $y$, and in the autonomous or periodic case we also prove that the attractor reduces to zero.

In what follows we will suppose that $\Omega$ and the functions involved satisfy the hypotheses of Theorem 2.3.

Let us begin with a remark in a simple case:

Consider the linear case with constant coeficients,

$$
\begin{array}{ll}
\Delta u+\sum_{i=0}^{n} 2 b_{i} u_{x_{i}}+c u=0 & \text { in } \Omega, \\
u(s, y)=0 & \text { for } y \in \partial \Omega^{\prime},
\end{array}
$$

When looking for bounded solutions of the type $u(s, y)=S(s) Y(y)$, it follows that $S(s)$ has to be a bounded solution of the ordinary differential equation $S^{\prime \prime}+$ $2 b_{0} S^{\prime}+c S=\mu S$ for some eigenvalue $-\mu$ of the operator $\Delta Y+\sum_{i=1}^{n} 2 b_{i} Y_{x_{i}}$ with Dirichlet boundary conditions in $\Omega^{\prime}$. In this case it holds that all bounded solutions $S(s)$ tend to zero as $s$ tends to $\infty$ if and only if

i) $c-\mu<0$ and $b_{0}$ arbitrary 
or

ii) $c-\mu>0$ and $b_{0} \neq 0$.

Let us now identify $\mu$. If we consider the problem

$$
\begin{array}{ll}
\Delta Y(y)+\sum_{i=1}^{n} 2 b_{i} Y_{x_{i}}(y)=-\mu Y(y) & \text { in } \Omega^{\prime}, \\
Y(y)=0 & \text { for } y \in \partial \Omega^{\prime},
\end{array}
$$

and we define $v(y)=\exp \left(\sum_{i=1}^{n} b_{i} x_{i}\right) Y(y)$ it follows that $v(y)$ satisfies

$$
\begin{array}{ll}
\Delta v(y)=\left(-\mu+\sum_{i=1}^{n} b_{i}^{2}\right) v(y) & \text { in } \Omega^{\prime}, \\
v(y)=0 & \text { for } y \in \partial \Omega^{\prime},
\end{array}
$$

So, we have that $\mu=-\lambda+\sum_{i=1}^{n} b_{i}^{2}$ where $\lambda(\lambda<0)$ are the eigenvalues of the Laplacian operator with Dirichlet boundary conditions in $\Omega^{\prime}$.

Therefore, if

$$
c+\lambda_{1}-\sum_{i=1}^{n} b_{i}^{2}<0,
$$

where $\lambda_{1}$ is the first eigenvalue of the Laplacian operator with Dirichlet boundary conditions in $\Omega^{\prime}$, all the bounded solutions of (4.1) of the type $u(s, y)=S(s) Y(y)$ tend to zero as $s$ tends to $\infty$.

Our aim in this section is to generalize the sufficient condition (4.2) for the general problem (2.9). Let us begin by stating some preliminary results.

Lemma 4.1. Let $w$ be a classical solution of

$$
\begin{array}{ll}
L[w]=\sum_{i, j=0}^{n} a_{i j}(x) w_{x_{i} x_{j}}+\sum_{i=0}^{n} 2 B_{i}(x) w_{x_{i}}+C(x) w \leq 0 & \text { in } \Omega, \\
w(s, y) \geq 0 & \text { for } y \in \partial \Omega^{\prime}, \\
w\left(s_{0}, y\right) \geq 0 & \text { for } y \in \Omega^{\prime}, \\
\lim _{s \rightarrow \infty} \inf \left\{w(t, y): t>s \text { and } y \in \Omega^{\prime}\right\} \geq 0 . &
\end{array}
$$

Then if $C(x) \leq 0$ for all $x \in \Omega$, it follows that $w(x) \geq 0$ for all $x \in \Omega$.

Proof: Let us assume the existence of $\bar{x} \in \Omega$ such that $w(\bar{x})<0$. From the hypotheses it follows that there exists $\bar{s}>s_{0}$ such that $w(s, y)>\frac{1}{2} w(\bar{x})$ for all $(s, y) \in[\bar{s}, \infty) \times \Omega^{\prime}$. This is in contradiction with the maximum principle applied to operator $L$ in the bounded domain $\left(s_{0}, \bar{s}\right) \times \Omega^{\prime}$ (see for instance Corollary 3.2 of $[7]$ ). 
Lemma 4.2. Let $v$ be a classical solution of

$$
\begin{array}{ll}
L[v]=\sum_{i, j=0}^{n} a_{i j}(x) v_{x_{i} x_{j}}+\sum_{i=0}^{n} 2 B_{i}(x) v_{x_{i}}+C(x) v=0 & \text { in } \Omega, \\
v(s, y)=0 & \text { for } y \in \partial \Omega^{\prime}, \\
\lim _{s \rightarrow \infty} v(s, y)=0 \text { uniformly on } y . &
\end{array}
$$

Then if $a_{00}(x) \leq A, B_{0}(x) \geq \bar{B}_{0}, C(x) \leq C<0$ for all $x \in \Omega$, and $\left|v\left(s_{0}, y\right)\right| \leq v_{0}$ for all $y \in \Omega^{\prime}$, it follows that

$$
|v(x)| \leq v_{0} \exp \left(m_{1}\left(s-s_{0}\right)\right) \quad \text { for all } x \in \Omega
$$

where $m_{1}:=\frac{-\bar{B}_{0}-\sqrt{\bar{B}_{0}^{2}-A C}}{A}<0$.

Proof: As $C<0$, there exists $m_{1}<0 \quad\left(m_{1}:=\frac{-\bar{B}_{0}-\sqrt{\bar{B}_{0}^{2}-A C}}{A}\right)$ such that $\psi(s)=v_{0} \exp \left(m_{1}\left(s-s_{0}\right)\right)$ is a solution of the ordinary differential equation $A \psi_{s s}+$ $2 \bar{B}_{0} \psi_{s}+C \psi=0$. Then, considering the linear operator $L$ it follows that

$$
\begin{aligned}
L[\psi] & =a_{00}(x) \psi_{s s}+2 B_{0}(x) \psi_{s}+C(x) \psi \\
& \leq A \psi_{s s}+2 \bar{B}_{0} \psi_{s}+C \psi=0
\end{aligned}
$$

and so that

$$
\begin{array}{ll}
L[\psi-v] \leq 0 & \text { in } \Omega, \\
(\psi-v)(s, y)=v_{0} e^{m_{1}\left(s-s_{0}\right)} \geq 0 & \text { for } y \in \partial \Omega^{\prime}, \\
(\psi-v)\left(s_{0}, y\right)=v_{0}-v\left(s_{0}, y\right) \geq 0 & \text { for } y \in \Omega^{\prime}, \\
\lim _{s \rightarrow \infty}(\psi-v)(s, y)=0 \text { uniformly on } y &
\end{array}
$$

and that

$$
\begin{array}{ll}
L[\psi+v] \leq 0 & \text { in } \Omega, \\
(\psi+v)(s, y)=v_{0} e^{m_{1}\left(s-s_{0}\right)} \geq 0 & \text { for } y \in \partial \Omega^{\prime}, \\
(\psi+v)\left(s_{0}, y\right)=v_{0}+v\left(s_{0}, y\right) \geq 0 & \text { for } y \in \Omega^{\prime}, \\
\lim _{s \rightarrow \infty}(\psi+v)(s, y)=0 \text { uniformly on } y &
\end{array}
$$

Therefore, by Lemma 4.1 it follows that $(\psi-v)(x) \geq 0$ for all $x \in \Omega$, and that $(\psi+v)(x) \geq 0$ for all $x \in \Omega$. Thus we have that

$$
|v(x)| \leq v_{0} \exp \left(m_{1}\left(s-s_{0}\right)\right)
$$

for all $x \in \Omega$, where $m_{1}:=\frac{-\bar{B}_{0}-\sqrt{\bar{B}_{0}^{2}-A C}}{A}<0$. 
Proposition 4.3. Let $u$ be a bounded classical solution of

$$
\begin{array}{ll}
\bar{L}[u]=\sum_{i, j=0}^{n} a_{i j}(x) u_{x_{i} x_{j}}+\sum_{i=0}^{n} 2 b_{i}(x) u_{x_{i}}+c(x) u=0 & \text { in } \Omega, \\
u(s, y)=0 & \text { for } y \in \partial \Omega^{\prime},
\end{array}
$$

where $a_{00}(x) \leq A$. Then, if there exists a function $\Phi(x) \in C^{2}(\Omega)$ and some constants $\eta, \bar{B}_{0}$ and $C<0$ such that

1. $\Phi(x) \geq \eta>0$, for all $x \in \Omega$.

2. $\lim \Phi(s, y)=\infty$ as $s$ tends to $\infty$, uniformly on $y$.

3. $\sum_{j=0}^{n} a_{0 j}(x) \Phi_{x_{j}}+b_{0}(x) \Phi \geq \bar{B}_{0} \Phi$ for all $x \in \Omega$.

4. $\bar{L}[\Phi] \leq C \Phi$ for all $x \in \Omega$.

5. $\lim \Phi(s, y) e^{m_{1}\left(s-s_{0}\right)}=0$ as $s$ tends to $\infty$, uniformly on $y$, where $m_{1}:=$ $\frac{-\bar{B}_{0}-\sqrt{\bar{B}_{0}^{2}-A C}}{A}<0$,

it follows that $\lim u(s, y)=0$ as $s$ tends to $\infty$, uniformly on $y$. of

Proof: Defining $v(x)=\frac{u(x)}{\Phi(x)}$ it follows that $v(x)$ is a bounded classical solution

$$
\begin{array}{ll}
L[v]=\sum_{i, j=0}^{n} a_{i j}(x) v_{x_{i} x_{j}}+\sum_{i=0}^{n} 2 B_{i}(x) v_{x_{i}}+C(x) v=0 & \text { in } \Omega, \\
v(s, y)=0 & \text { for } y \in \partial \Omega^{\prime}, \\
\lim _{s \rightarrow \infty} v(s, y)=0 \text { uniformly on } y, &
\end{array}
$$

where we note $B_{i}(x):=\sum_{j=0}^{n} a_{i j}(x) \frac{\Phi_{x_{j}}}{\Phi}+b_{i}(x)$ and $C(x)=\frac{\bar{L}[\Phi]}{\Phi}$.

Using now that $a_{00}(x) \leq A, B_{0}(x) \geq \bar{B}_{0}, C(x) \leq C<0$ for all $x \in \Omega$, by Lemma 4.2 it follows that

$$
|v(x)| \leq v_{0} \exp \left(m_{1}\left(s-s_{0}\right)\right) \quad \text { for all } x \in \Omega,
$$

and so by hypothesis 5$)$ that $\lim u(s, y)=0$ as $s$ tends to $\infty$, uniformly on $y$.

Now, thanks to Proposition 4.3 we are in position to generalize condition (4.2) for problems of the type (2.9). Let us begin with the linear case with coefficients depending on $x$.

Proposition 4.4. Let $b_{0}(x)$ be a bounded function on $\Omega$, and $c(x)$ such that there exists a constant $c<0$ with $c(x) \leq c<0$ for all $x \in \Omega$. Let $u$ be a bounded 
classical solution of

$$
\begin{array}{ll}
\bar{L}[u]=\sum_{i, j=0}^{n} a_{i j}(x) u_{x_{i} x_{j}}+\sum_{i=0}^{n} 2 b_{i}(x) u_{x_{i}}+c(x) u=0 & \text { in } \Omega, \\
u(s, y)=0 & \text { for } y \in \partial \Omega^{\prime},
\end{array}
$$

where $a_{00}(x) \leq A$ for all $x \in \Omega$. Then, it follows that $\lim u(s, y)=0$, exponentially, as $s$ tends to $\infty$, uniformly on $y$.

Proof: Let $b_{0}$ and $\bar{b}_{0}$ be such that $\bar{b}_{0} \leq b(x) \leq b_{0}$ for $x \in \Omega$ and define $\Phi(x)=\exp \left(\varepsilon\left(s-s_{0}\right)\right) \in C^{2}(\Omega)$ where we choose $\varepsilon$ in the range

$$
0<\varepsilon<\min \left(\frac{-b_{0}+\sqrt{b_{0}^{2}-A c}}{A}, \frac{-\left(b_{0}-\bar{b}_{0}\right)+\sqrt{\left(b_{0}-\bar{b}_{0}\right)^{2}-2 A c}}{2 A}\right) .
$$

It follows that

1. $\Phi(x) \geq 1>0$ for all $x \in \Omega$.

2. $\lim \Phi(s, y)=\infty$ as $s$ tends to $\infty$.

3. $\sum_{j=0}^{n} a_{0 j}(x) \Phi_{x_{j}}+b_{0}(x) \Phi=\left(a_{00}(x) \varepsilon+b_{0}(x)\right) \Phi \geq \bar{b}_{0} \Phi=: \bar{B}_{0} \Phi$ for all $x \in \Omega$.

4. $\bar{L}[\Phi]=\left(\varepsilon^{2} a_{00}(x)+2 \varepsilon b_{0}(x)+c(x)\right) \Phi \leq\left(\varepsilon^{2} A+2 \varepsilon b_{0}+c\right) \Phi=: C \Phi<0$ for all $x \in \Omega$.

5. $\lim \Phi(x) e^{m_{1}\left(s-s_{0}\right)}=\lim e^{\left(m_{1}+\varepsilon\right)\left(s-s_{0}\right)}=0$ as $s$ tends to $\infty$, uniformly on $y$, because $m_{1}+\varepsilon<0$. Here $m_{1}$ is defined as in Propositon 4.3.

So, we are in the hypotheses of Proposition 4.3 and thus $\lim u(s, y)=0$, exponentially (see (4.3)) as $s$ tends to $\infty$, uniformly on $y$.

The main result of this section is the following

Theorem 4.5. Let $\Omega$ and the functions $a_{i j}(x)$ and $f(p, z, x)$ satisfy the hypotheses of Proposition 2.6, and assume also that

i) The function $\partial f / \partial p_{0}$ is uniformly bounded for $x \in \Omega$ and $p$ and $z$ bounded.

ii) The function $f$ is of the form $f(0, z, x)=z C(z, x)$ where $C(z, x)$ satisfies that for all $M>0$ we have that $\sup \{C(z, x): x \in \Omega,|z| \leq M\}<0$.

Then if $u$ is a bounded classical solution of

$$
\begin{array}{ll}
S[u]=\sum_{i, j=0}^{n} a_{i j}(x) u_{x_{i} x_{j}}+f(\nabla u, u, x)=0 & \text { in } \Omega, \\
u(s, y)=0 & \text { for } y \in \partial \Omega^{\prime},
\end{array}
$$


where $a_{00}(x) \leq A$ for all $x \in \Omega$, it follows that $\lim u(s, y)=0$, exponentially, as $s$ tends to $\infty$, uniformly on $y$.

Remark: Observe that if $f$ does not depend on $x(f=f(p, z))$ then condition i) is automatically satisfied and condition ii) simply means that $f(0,0)=0$, $f_{z}(0,0)<0$ and $f(0, z) z<0$ for $z \neq 0$.

Remark: Observe that if condition ii) in Theorem 4.5 holds only for $M<\bar{M}$, then the claim remains true for the solutions $u$ such that the bound $|u|<\bar{M}$ is a-priori known.

Proof of Theorem 4.5: From the intermediate value theorem it follows that there exists $\xi(p, z, x) \in \mathbf{R}^{n+1}$ such that

$$
f(p, z, x)=f(0, z, x)+\sum_{i=0}^{n} \frac{\partial f(\xi, z, x)}{\partial p_{i}} p_{i}
$$

Define now

$$
2 \widetilde{b}_{i}(p, z, x)=\frac{\partial f(\xi, z, x)}{\partial p_{i}}, \quad i=0,1, \ldots, n
$$

Note that fixing $u$ we can consider $\widetilde{b}_{i}(\nabla u, u, x)$ and $C(u, x)$ as functions of $x$, i.e. $\tilde{b}_{i}(\nabla u, u, x)=b_{i}(x)$ and $C(u, x)=c(x)$. So, we can consider $u$ as a bounded classical solution of the linear problem (whose coefficients in fact depend on $u$ ):

$$
\begin{array}{ll}
\bar{L}[u]=\sum_{i, j=0}^{n} a_{i j}(x) u_{x_{i} x_{j}}+\sum_{i=0}^{n} 2 b_{i}(x) u_{x_{i}}+c(x) u=0 & \text { in } \Omega, \\
u(s, y)=0 & \text { for } y \in \partial \Omega^{\prime},
\end{array}
$$

where $a_{00}(x) \leq A$ for all $x \in \Omega$.

Finally, since $u$ is bounded and because of Proposition $2.6 \nabla u$ is bounded too in $\left[s_{0}+\delta, \infty\right) \times \Omega^{\prime}$ for some $\delta>0$, using i) and ii) it follows that we are in the hypotheses of Proposition 4.4 for a shifted domain $\Omega_{\delta}$. Thus $\lim u(s, y)=0$, exponentially as $s$ tends to $\infty$, uniformly on $y$.

Remark: A careful use of Proposition 2.6 in the proof above shows that the solution $u$ of (4.4) satisfies

$$
|u(s, y)| \leq C_{1}\left(\left\|u\left(s_{0}, y\right)\right\|_{\mathcal{C}^{\circ}\left(\Omega^{\prime}\right)}\right) \exp \left[\left(-C_{2}\left(\left\|u\left(s_{0}, y\right)\right\|_{\mathcal{C}^{\circ}\left(\Omega^{\prime}\right)}, \delta\right)\right)\left(s-s_{0}-\delta\right)\right]
$$

where $C_{1}$ and $C_{2}$ are positive functions which are nondecreasing with respect to $\left\|u\left(s_{0}, y\right)\right\|_{\mathcal{C}^{\circ}\left(\Omega^{\prime}\right)}$, and $\delta>0$ is arbitrary. This proves uniform asymptotic stability of the zero solution. In the autonomous or periodic cases considered in Section 3 this also implies that the global attractor reduces to zero. This remark also applies to Theorem 4.6 and Theorem 4.7 below. 
Condition ii) of Theorem 4.5 on the sign of the function $C(M)$ is very restrictive, although if it is natural in some contexts. The following two theorems give situations in which it can be weakened because of two facts. First the existence of a positive eigenvalue of the involved linear operator that translates the criticallity of the value $C(M)=0$ to a positive value $C(M)=\mu$. The second fact is the role that can be played by linear terms of first order (convection terms) if the coefficients are the gradient of some function. These theorems do not exhaust all the cases affected by these two facts, but they give examples on how to take profit from them.

Theorem 4.6. Let $\Omega$ and the functions $a_{00}(x)$ and $f(p, z, x)=\sum_{i=1}^{n} 2 b_{i}(y) u_{x_{i}}$ $+g(\nabla u, u, x)$ satisfy the hypotheses Proposition 2.6 where $b_{i}(y)=\partial B(y) / \partial x_{i}$, $i=1, \ldots, n$, with $B(y)$ being a bounded function in $\Omega^{\prime}$. Assume also that $g(p, z, x)$ satisfies the following hypotheses

i) The function $\partial g / \partial p_{0}$ is uniformly bounded for $x \in \Omega$ and $p$ and $z$ bounded.

ii) The function $g$ is of the form $g\left(0, p_{1}, \ldots, p_{n}, z, x\right)=z C\left(p_{1}, \ldots, p_{n}, z, x\right)$ where $C\left(p_{1}, \ldots, p_{n}, z, x\right)$ satisfies that for all $M>0$ and $N>0, \sup \left\{C\left(p_{1}\right.\right.$, $\left.\left.\ldots, p_{n}, z, x\right)-\Delta B(y)-|\nabla B(y)|^{2}+\lambda_{1}: x \in \Omega,\left|\left(p_{1}, \ldots, p_{n}\right)\right|<N,|z|<M\right\}<0$, where $\lambda_{1}(<0)$ is the first eigenvalue of the Laplacian operator with Dirichlet boundary conditions in $\Omega^{\prime}$.

Then, if $u$ is a bounded classical solution of

$$
\begin{array}{ll}
a_{00}(x) u_{s s}+\sum_{i=1}^{n} u_{x_{i} x_{i}}+\sum_{i=1}^{n} 2 b_{i}(y) u_{x_{i}}+g(\nabla u, u, x)=0 & \text { in } \Omega, \\
u(s, y)=0 & \text { for } y \in \partial \Omega^{\prime},
\end{array}
$$

when $a_{00}(x) \leq A$ for all $x \in \Omega$, it follows that $\lim u(s, y)=0$, exponentially, as $s$ tends to $\infty$, uniformly on $y$.

Remark: Observe that in the case $a_{00}(x)=1, b_{i}(y)=b_{i}$ and $g(\nabla u, u, x)=c u$ (that is problem (4.1)), conditions i) and ii) simply mean that $c+\lambda_{1}-\sum_{i=1}^{n} b_{i}^{2}<0$. which is exactly condition (4.2).

Proof of Theorem 4.6: From the intermediate value theorem it follows that there exists $\xi(p, z, x) \in \mathbf{R}$ such that

$$
g(p, z, x)=g\left(0, p_{1}, \ldots, p_{n}, z, x\right)+\frac{\partial g\left(\xi, p_{1}, \ldots, p_{n}, z, x\right)}{\partial p_{0}} p_{0}
$$

Define now

$$
\begin{aligned}
& 2 \widetilde{b}_{0}(p, z, x)=\frac{\partial g\left(\xi, p_{1}, \ldots, p_{n}, z, x\right)}{\partial p_{0}} \\
& \widetilde{c}(p, z, x)=C\left(p_{1}, \ldots, p_{n}, z, x\right) .
\end{aligned}
$$


Note again that fixing $u$ we can consider $\widetilde{b}_{0}(\nabla u, u, x)$ and $\widetilde{c}(\nabla u, u, x)$ as functions of $x$, i.e. $\widetilde{b}_{0}(\nabla u, u, x)=b_{i}(x)$ and $\widetilde{c}(\nabla u, u, x)=c(x)$. So, we can consider $u$ as a bounded classical solution of the linear problem (whose coefficients in fact depend on $u$ ):

$$
\begin{aligned}
& \bar{L}(u)=a_{00}(x) u_{s s}+\sum_{i=1}^{n} u_{x_{i} x_{i}}+2 b_{0}(x) u_{s}+\sum_{i=1}^{n} 2 b_{i}(y) u_{x_{i}}+c(x) u=0, \\
& u(s, y)=0, \\
& y \in \partial \Omega^{\prime},
\end{aligned}
$$

where $a_{00}(x) \leq A$ for all $x \in \Omega$.

Observe again that, since $u$ is bounded and because of Proposition $2.6 \nabla u$ is bounded too in $\Omega_{\delta}:=\left[s_{0}+\delta, \infty\right) \times \Omega^{\prime}$ for some $\delta>0$, and using i) and ii) there exist $b_{0}, \bar{b}_{0}$ and $c<0$ such that $\bar{b}_{0} \leq b_{0}(x) \leq b_{0}$ and $c(x)-\Delta B(y)-|\nabla B(y)|^{2}+\lambda_{1}<$ $c<0$, for the shifted domain $\Omega_{\delta}$.

Consider now $\Omega^{\prime \prime} \supset \supset \Omega^{\prime}$ such that $\bar{\lambda}(\bar{\lambda}<0)$, the first eigenvalue of the Laplacian operator with Dirichlet boundary conditions in $\Omega^{\prime \prime}$, satisfies that $c(x)-$ $\Delta B(y)-|\nabla B(y)|^{2}+\bar{\lambda}<c<0$. In these conditions we can define

$$
\Phi(x)=\exp \left(\varepsilon\left(s-s_{0}\right)-B(y)\right) \Lambda(y)
$$

for $x=(s, y) \in \Omega_{\delta}$, where $\Lambda(y)$ is such that

$$
\begin{array}{ll}
\Delta \Lambda(y)=\bar{\lambda} \Lambda(y) & \text { in } \Omega^{\prime \prime}, \\
\Lambda(y)=0 & \text { for } y \in \partial \Omega^{\prime \prime}
\end{array}
$$

so $\Lambda(y) \geq \Lambda>0$ in $\Omega^{\prime}$, for some $\Lambda$, and $\varepsilon$ is chosen in the range:

$$
0<\varepsilon<\min \left(\frac{-b_{0}+\sqrt{b_{0}^{2}-A c}}{A}, \frac{-\left(b_{0}-\bar{b}_{0}\right)+\sqrt{\left(b_{0}-\bar{b}_{0}\right)^{2}-2 A c}}{2 A}\right) .
$$

It follows that

1. $\Phi(x) \geq e^{-B} \Lambda>0$ for all $x \in \Omega_{\delta}$, where $B$ is an upper bound of $B(y)$.

2. $\lim \Phi(s, y)=\infty$ as $s$ tends to $\infty$, uniformly on $y$.

3. $\sum_{j=0}^{n} a_{0 j}(x) \Phi_{x_{j}}+b_{0}(x) \Phi=\left(a_{00}(x) \varepsilon+b_{0}(x)\right) \Phi \geq \bar{b}_{0} \Phi=: \bar{B}_{0} \Phi$.

4. $\bar{L}[\Phi]=\left(\varepsilon^{2} a_{00}(x)+2 \varepsilon b_{0}(x)+c(x)-\Delta B(y)-|\nabla B(y)|^{2}+\bar{\lambda}\right) \Phi$ $\leq\left(\varepsilon^{2} A+2 \varepsilon b_{0}+c\right) \Phi=: C \Phi<0$

5. $\lim \Phi(x) e^{m_{1}\left(s-s_{0}\right)}=\lim e^{\left(m_{1}+\varepsilon\right)\left(s-s_{0}\right)-B(y)} \Lambda(y)=0$ as $s$ tends to $\infty$, uniformly on $y$, because $m_{1}+\varepsilon<0$ ( $m_{1}$ is defined as in Proposition 4.3).

So, we are in the hypotheses of Proposition 4.3 for the shifted domain $\Omega_{\delta}$ and thus $\lim u(s, y)=0$, exponentially, as $s$ tends to $\infty$, uniformly on $y$. 
Theorem 4.7. Let $\Omega$ and $f(p, z, x)=\sum_{i=0}^{n} 2 b_{i}(x) u_{x_{i}}+g(\nabla u, u, x)$ satisfy the hypotheses of Proposition 2.6 with $b_{0}(x)$ being bounded in $\Omega$. Assume that there exist a bounded function $B(x), x \in \Omega$, such that $b_{i}(x)=\partial B(x) / \partial x_{i}$, $i=0,1, \ldots, n$. Assume also that $g(p, z, x)$ is a function of the form $g(p, z, x)=$ $z C(p, z, x)$ where $C(p, z, x)$ satisfies that for all $M>0$ and $N>0$ we have that $\sup \left\{C(p, z, x)-\Delta B(y)-|\nabla B(y)|^{2}+\lambda_{1}: x \in \Omega,|p|<N,|z|<M\right\}<0$, where $\lambda_{1}(<0)$ is the first eigenvalue of the Laplacian operator with Dirichlet boundary conditions in $\Omega^{\prime}$. Then, if $u$ is a classical bounded solution of

$$
\begin{array}{ll}
\Delta u+\sum_{i=0}^{n} 2 b_{i}(x) u_{x_{i}}+g(\nabla u, u, x)=0 & \text { in } \Omega, \\
u(s, y)=0 & \text { for } y \in \partial \Omega^{\prime},
\end{array}
$$

it follows that $\lim u(s, y)=0$, exponentially, as $s$ tends to $\infty$, uniformly on $y$.

Proof of Theorem 4.7: This proof is analogous to the proof of Theorem 4.6 by using the function $\Phi(x)=\exp \left(\varepsilon\left(s-s_{0}\right)-B(x)\right) \Lambda(y), x \in \Omega_{\delta}$.

\section{References}

[1] Berestycki, H.; L. Nirenberg: Some qualitative properties of solutions of semilinear elliptic equations in cylindrical domains. Analysis, et cetera. (P.H. Rabinowitz and E. Zchuder, Eds.), Academic Press, 1990, p. 115-164.

[2] Brunovský, P.; X. Mora; P. Polácik; J. Solà-Morales: Asymptotic behavior of solutions of semilinear elliptic equations on an unbounded strip. Acta Math. Univ. Comenianae LX 2, 1991, p. 163-183.

[3] Calsina, A.; X. Mora; J. Solà-Morales: The dynamical approach to elliptic problems in cylindrical domains, and a study of their parabolic singular limit. J. Differential Equations 102, 1993, p. 244-304.

[4] Chen, X.Y.; H. Matano; L. Véron: Singularités anisotropes d'équations élliptiques sémilinéaires dans le plan. C.R. Acad. Sci. Paris 303, 1986, p. 963-966. Anisotropic singularities of solutions of nonlinear elliptic equations in $\mathbf{R}^{2}$. J. Funct. Anal.83, 1989, p. 50-97.

[5] Díaz, J.I.; O.A. Oleinik: Nonlinear elliptic boundary-value problems in unbounded domains and the asymptotic behaviour of its solutions. C. R. Acad. Sci. Paris 315, 1992, p. 787-792.

[6] Flavin, J.N.; R.J. Knops; L.E. Payne: Asymptotic behaviour of solutions to semi-linear elliptic equations on the half-cylinder. ZAMP 43, 1992, p. 406-421.

[7] Gilbarg, D.; N.S. Trudinger: Elliptic partial differential equations of second order (1st edition). Springer-Verlag, 1977.

[8] Hale, J.: Asymptotic behavior of dissipative systems. American Mathematical Society, 1988.

[9] Kirchgässner, K.: Wave solutions of reversible systems and applications. $J$. Differential Equations 45, 1982, p. 113-127. 
[10] Ladyženskaja, O.A.; N.N. Ural'ceva: Equations aux dérivées partielles de type élliptique. Dunod, 1968.

[11] Mielke, A.: Inertial manifolds for elliptic problems in infinite cylinders. Preprint. University of Stuttgart, 1990.

[12] Vega, J.: The asymptotic behavior of the solutions of some semilinear elliptic equations in cylindrical domains. J. Differential Equations 102, 1993, p. $119-152$. 